\title{
Information reduction, internal transformations, and task difficulty
}

\author{
BRUCE A. AMBLER \\ University of Texas at Arlington, Arlington, Texas 76019 \\ SEBASTIANO A. FISICARO \\ Western Kentucky University, Bowling Green, Kentucky 42101 \\ and \\ ROBERT W. PROCTOR \\ Auburn University, Auburn, Alabama 36830
}

\begin{abstract}
Three experiments investigated the effects of information reduction and number of internal transformations in determining task difficulty. The primary dependent variable examined was pupil dilation. In Experiment 1, pupil size was found to increase with increases in amount of information reduction. However, number of internal transformations was confounded with information reduction. In Experiment 2, when information reduction was held constant, it was found that increasing the number of internal transformations resulted in increased pupil size. In Experiment 3, when number of internal transformations was held constant but amount of information reduction was varied, no differences in pupil dilation were found. It was concluded that the usual increase in task difficulty observed with increases in information reduction is due to increases in the number of internal transformations that must be performed.
\end{abstract}

Posner (1964) introduced the concept of information reduction as one factor influencing the difficulty of mental tasks. He defined information reduction as the differences between the amount of stimulus information ( $\log _{2}$ of the number of alternative stimuli) and the amount of response information $\left(\log _{2}\right.$ of the number of possible response alternatives). ${ }^{1}$ Posner found that the task difficulty (as indicated by an error rate measure) increased monotonically with the amount of information reduction.

Several authors (Garner, 1974; Keele, 1970) have interpreted the effect of information reduction on task difficulty as being due to additional internal transformations that must be performed on the stimulus to arrive at a response. However, there appears to be no critical evidence that specifically links task difficulty to these additional internal transformations. Thus, the primary purpose of the present study was to independently vary information reduction and number of internal transformations to determine whether number of internal trnasformations is the cause of the commonly observed information reduction effect.

Pupil dilation was employed as a dependent variable in addition to error rate. This was done for three basic reasons: First, a large amount of evidence suggests that pupillary dilations reflect the amount of mental effort,

Requests for reprints should be sent to Bruce A. Ambler, Department of Psychology, University of Texas at Arlington, Arlington, Texas 76019. or processing capacity, required to perform a task (Kahneman, 1973). Second, pupil size has been shown to increase with task difficulty (e.g., Bradshaw, 1968; Hess \& Polt, 1964). And third, there is some evidence that suggests that pupil size is more sensitive than error rate to differences in task difficulty (Ambler, Fisicaro, \& Proctor, 1976).

\section{EXPERIMENT 1}

Experiment 1 was essentially a replication of the Posner (1964) experiment using pupil dilation as a dependent variable. The primary purpose of the experiment was to validate the pupil dilation measure as being sensitive to the levels of difficulty in information reduction tasks. Thus, in Experiment 1 no attempt was made to control for number of internal transformations.

\section{Method}

Subjects. The subjects were four graduate students and eight undergraduate volunteers from an introductory course in psychology.

Stimulus materials. Sixty-eight two-digit numbers between 0 and 99 were selected from a table of random numbers with the constraint that no number could be composed of double digits (e.g., 22). These were randomly divided into four lists of 17 numbers each. Stimuli were tape recorded in a male voice as two separate digits (e.g., "four, eight" or "zero, five"). The intertrial interval (ITI) was 15 sec.

Apparatus. The apparatus is the same as that used by Ambler et al. (1976). Stimuli were presented to subjects through stereo 
headphones from a tape recorder. Pupil dilations were recorded on videotape and were measured at a later time.

Conditions. Four conditions were examined. In the list condition, subjects were instructed to repeat the two digits in the same order that they heard them. The reverse condition required subjects to repeat the two digits in the reverse order. The add condition required adding the two digits and responding with the sum. In the classify condition, the first digit was to be judged as being high (5-9) or low (0-4). The second digit was to be judged as being odd or even. Subjects responded " $A$ " if the digit pair was high and odd or low and even; otherwise, they responded "B."

The amount of stimulus information was 6.6 bits for each task. This computation was made on the assumption that subjects believed that all 100 two-digit numbers were possible even though double-digit numbers were excluded. Response information in the list and reverse conditions was also 6.6 bits because in these conditions the subject could respond with any of 100 different digit pairs. Thus, there was zero information reduction in these two conditions. Theoretically, the sums in the add condition could take on values from 0 to 18 , resulting in response information of 4.1 bits and an information reduction of 2.5 bits. In the classify condition, there were only two possible responses (1.0 bit of response information). Therefore, the amount of information reduction in the classify condition was 5.6 bits.

The tasks also differed in the number of transformations required. All tasks required the encoding of two digits and the output of a response. However, the reverse task also required the use of an extra transformation to reverse the two digits, the add task involved use of the set of transformations required for addition, and the classify task involved the transformations required to make high-low, even-odd, and A-B decisions.

Design. The experiment was a within-subjects one-factor design, the factor consisting of the four tasks. The tasks were run in separate blocks, with order counterbalanced by a Latin square technique. Thus, there were four different orders and three subjects per order.

Procedure. After the subject was seated, instructions and examples were given for the first task and all questions were answered. It was emphasized that the eye had to be kept still and fixated in the center of the camera lens during the trials. The apparatus was then started and the subject received 17 trials in each of the four tasks. The subject's accuracy on all trials was recorded by the experimenters.

After the subject left, the videotape was played back and pupil dilation measures were made using a calibrated transparent grid placed over the video monitor. Data were collected from the last 12 correct trials in each of the four conditions. Thus, the first few. trials were treated as practice. Within each correct trial, three pupil dilation measures were taken in the following manner. Two baseline measures were taken during the 15 -sec ITI. The first was taken $8 \mathrm{sec}$ after the presentation of the previous stimulus and the second was taken $5 \mathrm{sec}$ after the first. For all conditions, pupil dilation appeared to reach a maximum within $1 \mathrm{sec}$ after stimulus presentation and remain constant for a short period of time before contracting. Since previous research also indicates pupil dilation reaches maximum within $1 \mathrm{sec}$ (Kahneman, 1973), trial measures were taken at this point.

For each subject, one mean was computed for the 24 baseline measures and one mean was computed for the 12 trial measures in each condition. A difference score was obtained by subtracting the mean baseline from the respective trial mean. This was done for each of the four conditions, and an analysis of variance was performed on the mean difference scores. The number of errors was also recorded for each subject, and an analysis of variance was performed on error rates.

\section{Results and Discussion}

The mean percentage of errors and the mean differ-
Table 1

Mean Difference in Pupil Size (in Millimeters) and Mean Percent Errors as a Function of Task Requirements, Experiment 1

\begin{tabular}{lccrc}
\hline & List & Reverse & Add & Classify \\
\hline Pupil Dilation & .30 & .32 & .39 & .47 \\
Error Rate & .83 & .83 & 2.50 & 10.00 \\
\hline
\end{tabular}

ence scores for pupil size are presented in Table 1. The analysis of variance on the pupil dilation data was significant $[\mathrm{F}(3,33)=5.08, \mathrm{p}<.01, \mathrm{MSe}=.016]$. Pupil dilation was obviously directly related to the amount of information reduction. A Scheffé test was used to compare the list and reverse tasks (equal in bits of information reduction). Even though they did not differ significantly ( $p>.05), 9$ of the 12 subjects did exhibit greater pupillary dilations for the reverse task.

An analysis of variance was also performed on the error rates of 10 subjects. The error scores for the other two subjects were lost due to experimenter error. The analysis of variance was significant $[\mathrm{F}(3,27)=5.97$, $\mathrm{p}<.01, \mathrm{MSe}=32.1]$. The error rates for the list and reverse tasks clearly did not differ. As with pupil dilation, error rate was also directly related to the amount of information reduction.

The results of Experiment 1 were very similar to those reported by Posner (1964). Thus, pupil dilation does appear to be sensitive to differences in task difficulty in information reduction tasks. Error rate and pupil dilation increased as information reduction increased. However, number of internal transformations might also account for the obtained differences. The one possible exception to this was the failure to find a difference between the list and reverse tasks, which differed in the number of required internal transformations. However, since it is not clear that subjects actually had to perform a reversal transformation (i.e., they may have echoed back the second digit and then retrieved the first), and the reverse task did result in greater pupil dilation for 9 of 12 subjects, a rejection of the hypothesis that internal transformations influence pupil dilation seems premature.

\section{EXPERIMENT 2}

As noted previously in Experiment 1, differences in information reduction and number of internal transformations were confounded across conditions. Experiment 2 was designed to hold information reduction constant while varying the number of internal transformations required to complete the task.

\section{Method}

Subjects. The subjects were four advanced undergraduate psychology majors and 14 undergraduate volunteers from an introductory psychology course.

Stimulus materials. Three lists of 10 one-digit numbers (0-9) were constructed by randomly selecting the order of each 
list independently of the other lists. Following this, for each list, five one-digit numbers were randomly selected and added to the end of the list. The three 15-digit lists were recorded in a male voice with a 5-sec ITI.

Apparatus. The apparatus was the same as in Experiment 1.

Conditions. The three conditions were high-low (H-L), evenodd (E-O), and Classify. In the H-L condition, if a digit was high $(\geqslant 5)$ subjects responded $A$; if it was low $(\leqslant 4)$, they responded B. In the E-O condition, if the digit was even the response was $\mathbf{A}$; if odd, it was B. Under classify instructions, subjects were required to make both high-low and even-odd judgments about the single digit. If the digit was high and odd or low and even, they responded A; otherwise, they said $B$.

Since the number of possible stimulus items was 10 (3.3 bits) and there were two response alternatives $(1.0 \mathrm{bit})$ in each condition, the amount of information reduction was the same (2.3 bits) for all three conditions. Also, the number of transformations was the same for the H-L and E-O conditions. However, the classify task required at least one more transformation than either of the other two tasks.

Design. The experiment was a single-factor within-subjects design. Latin squares randomization resulted in three different orders of the three conditions, with six subjects tested on each order. Orthogonal comparisons were used to analyze the data.

Procedure. The procedure was basically the same as the one reported in Experiment 1, with the exception that subjects were given 15 trials on each of the three tasks. Pupil dilation measures were taken on the first 10 correct trials with the same procedure used in Experiment 1. This resulted in 20 baseline measures and 10 trial measures per condition for each subject. The differences between mean baseline and mean trial pupil sizes were computed for each subject in each condition. These mean differences were used in the analysis. Error rates were also computed and analyzed.

\section{Results and Discussion}

The mean difference scores for the pupil size and mean percent errors are presented in Table 2. Orthogonal comparisons produced the following results for pupil dilation: There was no significant difference between the $\mathrm{H}-\mathrm{L}$ and $\mathrm{E}-\mathrm{O}$ conditions $[\mathrm{F}(1,34)<1.0, \mathrm{MSe}=.010]$. However, these two tasks did differ significantly from the classify task $[\mathrm{F}(1,34)=6.49, \mathrm{p}<.025, \mathrm{MSe}=.010]$. Orthogonal comparisons were also performed on error scores. There was no difference between the $\mathrm{H}-\mathrm{L}$ and E-O conditions $[\mathrm{F}(1,34)<1.0, \mathrm{MSe}=27.1]$, and no difference between those two tasks and the classify task $[\mathrm{F}(1,34)<1.0, \mathrm{MSe}=27.1]$.

When the H-L and E-O conditions were compared, no difference in pupil size was observed. This indicates that the different transformation involved in these two conditions did not have differential effects on pupil size. However, when information reduction was equated but number of transformations was varied (H-L and E-O vs.

Table 2

Mean Difference in Pupil Size (in Millimeters) and Mean Percent Errors as a Function of Task Requirements, Experiment 2

\begin{tabular}{lrrc}
\hline & H-L & E-O & Classify \\
\hline Pupil Dilation & .33 & .33 & .41 \\
Error Rate & 2.22 & 1.11 & 5.00 \\
\hline
\end{tabular}

Classify) a difference in pupil size was observed. This result indicates that differences in pupil size can be obtained even when the amount of information reduction does not vary.

Furthermore, the difference in pupil size was observed in the absence of a significant difference in error rate. At least two possible explanations exist for this. One is that error rate is an index of variations in information reduction. If this were true, then the lack of a difference in error rate between tasks in Experiment 2 would be expected. Another possibility is that, when error rate is low, it is merely less sensitive than pupil dilation to the variables presently being studied.

\section{EXPERIMENT 3}

Even though differences in pupil size were obtained when information reduction remained the same (Experiment 2), the possibility still exists that information reduction influences pupil dilation. The purpose, then, of Experiment 3 was to hold number of internal transformations constant while varying the amount of information reduction required to perform a task. Additionally, if error rate measures something specific to information reduction, then this experiment should produce differences in error rate between tasks.

\section{Method}

Subjects. The subjects were nine undergraduate volunteers from an introductory psychology class and one advanced student in psychology.

Stimulus materials. One list of 15 two-digit numbers was compiled using the procedure described in Experiment 1, and one list of 15 one-digit numbers was formed using the technique described in Experiment 2. Stimuli were recorded in a male voice with a 15 -sec ITI.

Apparatus. The apparatus was the same as that used in Experiment 1.

Conditions. Subjects were instructed to classify the numbers in both of the lists. The classify condition that consisted of twodigit numbers was the same as the one described in Experiment 1; the classify condition consisting of one-digit numbers was the same as the one described in Experiment 2.

As noted in Experiment 1, the two-digit classify condition contained 5.6 bits of information reduction; and, as described in Experiment 2, the one-digit classify condition involved 2.3 bits of information reduction.

Design. Within-subjects one-factor designs were used. Half of the subjects received the two-digit classify condition first, and half were given the one-digit classify condition first.

Procedure. The procedure was the same as that reported in Experiment 1, with the exception that each condition had 15 stimulus items. The same procedure for taking pupil dilation measurements and error scores was used in this experiment.

\section{Results and Discussion}

Mean differences in pupil size and mean error rates are shown in Table 3. Both analyses of variance resulted in nonsignificance $[\mathrm{F}(1,9)<1.0, \mathrm{MSe}=.005$ for the pupil dilation data; $F(1,9)=1.98, p>.05, \mathrm{MSe}=22.8$ for the error data]. Thus, neither pupil dilation nor error rate was related to amount of information reduction. 
Table 3

Mean Difference in Pupil Size (in Millimeters) and Mean Percent Errors as a Function of Task Requirements, Experiment 3

\begin{tabular}{lcc}
\hline & \multicolumn{2}{c}{ Classify } \\
\cline { 2 - 3 } & One Digit & Two Digit \\
\hline Pupil Dilation & .30 & .29 \\
Error Rate & 4.00 & 7.00 \\
\hline
\end{tabular}

The results indicate that when number of internal transformations is held constant, no factors correlated with information reduction have an effect on task difficulty. Thus, the combined results of Experiments 2 and 3 indicate that information reduction itself is not related to task difficulty. Rather, it appears that the number of internal transformations, which is usually confounded with information reduction, accounts for differences in task difficulty. Additionally, the present results support the second explanation offered in Experiment 2 for the lack of a significant difference in error rate. That is, as Ambler et al. (1976) suggest, pupil dilation seems to be more sensitive than error rate to differences in task difficulty.

\section{REFERENCES}

characteristics of primary-secondary message interference in a dichotic listening task. Memory \& Cognition, 1976, 4, 709-716.

BRADshaw, J. L. Pupil size and problem solving. Quarterly Journal of Experimental Psychology, 1968, 20, 116-122.

GARNER, W. R. The processing of information and structure. Potomac. Md: Erlbaum, 1974.

Hess, E. H., \& Polt, J. M. Pupil size in relation to mental activity during simple problem-solving. Science, 1964, 143, 1190-1192.

Kahneman, D. Attention and effort. Englewood Cliffs, N.J: Prentice-Hall, 1973.

KeELE, S. W. Effects of input and output modes on decision time. Journal of Experimental Psychology, 1970, 85. 157-164.

POSNER, M. I. Information reduction in the analysis of sequential tasks. Psychological Review', 1964, 71, 491-504.

\section{NOTE}

1. This manner of computing amount of information assumes that each alternative has an equal probability of occurrence. If this is not the case, then the following formula must be used in calculating amount of information:

$$
\sum_{i=1}^{n} P_{i} \times \log _{2}\left(1 / P_{i}\right)
$$

where $n$ is the number of alternatives and $P_{i}$ refers to the probability of occurrence of the ith alternative.

(Received for publication August 1, 1977.) 